\title{
MOTOR INNERVATION OF MYASTHENIC MUSCLES RELATED TO AGE
}

$\mathrm{SIR}$,-Various changes in the motor innervation of myasthenic muscles have been recorded. Two main abnormal patterns of motor end-plates have been found: (1) expanded endings sometimes formed by several motor arborisations, with marked irregularity of axoplasmic expansions; and (2) elongated endings with lack of side branching. ${ }^{1-3}$ In addition, an increased collateral ramification of motor axons was occasionally noticed. ${ }^{4,3}$

In an attempt to understand the functional significance of these changes, we compared their incidence with various clinical data, including the sex and age of patients, the severity and apparent duration of the disease, and histological changes in the muscle.

Intramuscular nerve-fibres and motor-endings were studied on muscle biopsies, vitally stained with methyleneblue, obtained from 45 myasthenic patients. The incidence and proportion of abnormal motor endings were recorded, and the collateral branching of motor nervefibres was estimated by the terminal innervation ratio (T.I.R.): number of muscle-fibres innervated by a given number of subterminal motor-axons.

Expanded motor endings were found in 38 biopsies, without any demonstrable relationship to age, sex, or

MOTOR-DNNERVATION PATTERN IN RHLATION TO AGE OF PATHENTS

\begin{tabular}{|c|c|c|c|c|c|c|}
\hline \multirow{2}{*}{\multicolumn{3}{|c|}{ Pattern }} & \multirow{2}{*}{$\begin{array}{c}\text { No, } \\
\text { of } \\
\text { patients }\end{array}$} & \multicolumn{2}{|c|}{ Age (yr.) } & \multirow{2}{*}{ Significance } \\
\hline & & & & Mean & S.D. & \\
\hline \multicolumn{3}{|c|}{ Elongated motor endirgs: } & & & & \multirow{4}{*}{$t=1.906 \mathrm{P} \approx 0.05$} \\
\hline Present & .. & .. & 26 & $42 \cdot 15$ & $19-4$ & \\
\hline Absent & .. & $\ldots$ & 19 & $52-11$ & $13 \cdot 8$ & \\
\hline \multicolumn{6}{|l|}{ T.I.R.: } & \\
\hline Normal & .. & .. & 38 & $43-4$ & $7 \cdot 2$ & \multirow{2}{*}{$t=2.76 \quad t<0-01$} \\
\hline Increased & , & .. & 7 & $62 \cdot 3$ & $17 \cdot 7$ & \\
\hline
\end{tabular}


severity of the disease, or to the presence of histological alterations of the muscle tissue. Elongated motor endings were present in 26 biopsies in a variable proportion of from 2 to $78 \%$. No correlation was found between the incidence of this change and the severity of the disease, but there was a greater frequency of elongated motor endings in younger patients (see accompanying figure). Comparison of biopsies with and without elongated motor endings shows that the mean age of patients whose biopsies contained such nerve endings is significantly lower than in the other group (see accompanying table). The T,I,R. was increased in 7 biopsies, all from patients over the age of 50 (see figure). No relation was found between this change and clinical or histological data. There is a significant difference between the mean age of the groups of patients in whose biopsies a normal or increased T.I.R. was observed (see table).

These morphological differences in relation to age suggest a chronological evolution of the motor-innervation pattern in myasthenic muscles. Elongation of motor endings, commoner in younger patients, could be a primary malformation of the myoneural junction, as previously assumed ("dysplastic" end-plates)., ${ }^{1,2}$ It could also represent the earliest change produced by an autoimmune

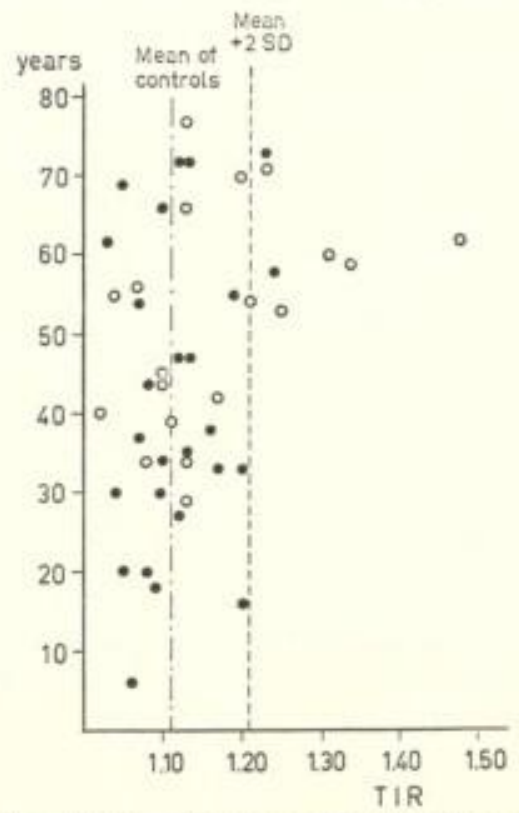

Distribution of T.I.R. and incidence of elongated motor endings in relation to age.

Biopsies without elongated motor endings.

- Biopsies with elongated motor endings. 
process affecting the motor end-plate. ${ }^{7}$ Distal branching of motor axons forming expanded or multiple motor arborisations could represent a compensation to impaired neuromuscular transmission, similar to the axonal changes obtained by local application of botulinum toxin." Collateral branching of motor nerve-fibres increasing the T.I.R. and indicating reinnervation of adjacent denervated musclefibres \$, appears to be a late feature in myasthenia, taking place only in elderly patients whatever the apparent duration or the severity of their disease.

Another possibility could be that the presence of elongated motor endings characterises a type of myasthenia occurring preferentially in a younger age-group than other types of myasthenic syndromes, some of them being related to denervation and affecting older subjects.

Free University of Brussels,

Department of Neurology,

Medical Clinic,

Hopital Brugmann, Brussels, Belgium.

C. CoẼrs.

1. Coêrs, C., Woolf, A. L. The Innervation of Muscle: a Biopsy Study. Oxford, 1959.

2. Coêrs, C., Desmedt, J. E. Acta neurol, belg, 1959, 59, 539.

3. Coërs, C in Progressive Muskeldystrophie, Myotonic, Myasthenie (edited by E. Kuhn); p. 325. Berlin, 1966.

4. Coèrs, C., Telerman-Toppet, N., Gerard, J. M. Archs Nesrol., Chicago, 1973, 29, 210, 215.

5. Woolf, A. L., Coèrs, C. in Disorders of Voluntary Muscles (edited by J. N. Walton). London, 1973.

6. Coèrs, C. Acta neirol. belg. 1955, 55, 741.

7. Engel, A. G., Tsuitihata, M., Lindstrom, Ji, Lennon, V. A. Conference on Myasthenia Gravis, abstr. no, 5, New York, May 30. 1975.

8. Duchen, L. W. J. Neterol. Neurosurg. Psychiat, 1970, 33, 40. 